\title{
'Characterization and outcome of packed red blood cells transfusion in critically ill children in PICU in Sohag University hospital
}

\author{
Hossam K. ALI ${ }^{1}$,M.B,B.Ch.,Shaimaa M. Mahmoud ${ }^{2}$,MD,.Alzahraa Alsayed Ahmed \\ ${ }^{3}, \mathrm{MD}$.
}

1:M.B,B.Ch.,Demonestrator of Pediatrics,Sohag University Hospital.2: MD,Lecturer of Pediatrics ,Sohag University Hospital. 3:MD,Professor of Pediatrics ,Sohag University Hospital.

\begin{abstract}
Red blood cell transfusions are a common therapy in critically ill anemic children ,a packed cell product is the component of choice for replacement therapy during RBC loss and sporadic transfusion therapy.

The decision to transfuse must be based on an assessment of the risks of anemia versus the risks of transfusion. In addition to the individual assessment of any symptomatic anemic child, the duration of anemia must be taken into consideration as well as the extent of trauma or surgery and the probability of blood loss and coexisting conditions such as impaired pulmonary function and inadequate cardiac output. ${ }^{(\mathbf{1 - 3})}$

This study was a prospective observational study conducted for one year in Pediatric Intensive Care Unit (PICU) of SohagUniversity Hospital ,a tertiary health center which provide specialized care to critically ill infants and children with about $\mathbf{4 0 0}$ admissions yearly.Patients was divided to two groups according to arterial blood pressure.

Stable critically ill: not hypotensive (mean arterial pressure not less than $\mathbf{2}$ standard deviations below the mean for age), or no cardiovascular support for at least two hours prior to enrollment.

Unstable critically ill: hypotensive (mean arterial pressure less than $\mathbf{2}$ standard deviations below the mean for age), or there is cardiovascular support for at least two hours prior to enrollment, this includes patients with heart failure.

Packed RBCS transfusion following arestrictive blood transfusion strategywas used. Stable critically ill patients: received blood transfusion only if hemoglobin concentration falls below $7 \mathrm{gm} / \mathrm{dl}$.

Unstable critically ill patients: received blood transfusion according to clinical judgment. ${ }^{(1,4)}$

Short term outcome till discharge including (mortality or improvement) and duration of ICU stay was observed and recorded.

Our study showed that respiratory diseases were the main risk factor that increase the duration of PICU stay ( $p$ value $\mathbf{0 . 0 0 1}$ ), while suffering from hemodynamic unstablity was the main risk factor for death in our PICU (P value 0.02) .a restrictive blood transfusion strategy was safe in pediatric patients whose condition was stable in PICU, with no effect on mortality or duration of admission in PICU andthe studywas not able to highlight a cause-effect relationship between RBC transfusions and outcomes in critically ill children.
\end{abstract}

Key words:Packed RBCs ,Transfusion, Critically ill, Pediatric ICU 


\section{Introduction}

Red blood cell transfusions are a common therapy in critically ill anemic children ,a packed cell product, which comprises the

sedimented or centrifuged RBCs from one unit of single-donor whole blood, is the component of choice for replacement therapy during $\mathrm{RBC}$ loss (e.g., owing to surgery or trauma) and sporadic transfusion therapy.

The decision to transfuse must be based on an assessment of the risks of anemia versus the risks of transfusion. in addition to the individual assessment of any symptomatic anemic child, the duration of anemia must be taken into consideration as well as the extent of trauma or surgery andthe probability of blood loss and coexisting conditions such as impaired pulmonary function and inadequate cardiac output. ${ }^{(\mathbf{1 - 3})}$

The transfusion strategy in critically ill children remains controversial and has generated much research and debate.

\section{Aim of the work}

To study the epidemiology and short term outcome of packed RBCS transfusion in critically ill children admitted to PICU and to determine the incidence,indications anda strategy to usefor pRBCs transfusion in critically

\section{Patients and Methods}

ill children admitted to PICU.

This study was a prospective observational study conducted for one year in Pediatric Intensive Care Unit (PICU) of SohagUniversity Hospital ,a tertiary health center which provide specialized care to critically ill infants and children with about 400 admissions yearly. Patients was divided to two groups according to arterial blood pressure.

Stable critically ill: Patients were considered stable if they were not hypotensive (mean arterial pressure not less than $\mathbf{2}$ standard deviations below the mean for age), or no cardiovascular support for at least two hours prior to enrollment.

Unstable critically ill: Patients were considered unstable if they were hypotensive (mean arterial pressure less than 2 standard deviations below the mean for age), or there is cardiovascular support for at least two hours prior to enrollment, this includes patients with heart failure.

Packed RBCS transfusion following a restrictive blood transfusion strategywas used

Stable critically ill patients : received blood transfusion only if hemoglobin concentration falls below 7 $\mathrm{gm} / \mathrm{dl}$.

Unstable critically ill patients: received blood transfusion according to clinical judgment(Hemoglobin level and other determinants of RBC transfusion may play a role, like acute blood loss and $\mathrm{ScvO} 2(<70 \%)$, but the usefulness of these markers to drive goaldirectedtransfusion therapy remains to be determined) ${ }^{(\mathbf{1 , 4})}$

Packed red blood cells was (5) given in dose of $\mathbf{1 0} \mathrm{ml} / \mathrm{kg}$ of body weight

\section{Study size and sampling}

\subsection{Study population}

Our study was conducted on $\mathbf{1 9 0}$ critically ill children of total $\mathbf{3 9 2}$ critically ill child admitted to PICU (48.46\% of admissions).

\subsection{Patient selection}

Inclusion criteria: any critically ill child received $\mathrm{pRBCs}$ transfusion aged from one month to 18 years admitted to PICU and pediatric Emergency unit during the study period. 


\section{Exclusion criteria:}

Patients with cyanotic congenital heart diseases , acute blood loss, platelet dysfunction, hemolytic anemia, survival for less than 24 hours .

\section{Ethical consideration}

Approval of Sohag faculty of medicine research ethics committee was obtained and verbal consent was obtained from guardians.

\section{Patient handling and data collection during the study} Patient stabilization and monitoring according to PICU Protocols.after patient stabilization medical history was collected from patient relatives . These include demographic data; severity of illness parameters. Diagnosis on admission was taken from patient sheet as defined by caring medical attendant.

Complete clinical examination was conducted. importantlyhemodynamic stability, vital signs and cardiovascular system examination. hemodynamic stability was frequently checked by pulse rate and volume evaluation (rates for age were judged with specific centile tables), capillary refill time determination and regular systematized blood pressure measurement, blood pressure measurements were compared with normal values. Short term outcome till discharge including (mortality or improvement) and duration of ICU stay was observed and recorded.

Investigators did not intervene in patient management, which was provided by attending physician using standard protocols.

\section{Statistical analysis:}

\section{A. descriptive statistics which included:}

Demographic characteristics,indications for admission to PICU,clinical features of studied critically ill patients in PICU,duration of admission and outcome of studied critically ill patients in PICU, prevalence, frequency and indications of PRBCs transfusion of studied critically ill patients in PICU.

Comparison between transfused cases with non-transfused cases as regards age, diagnosis ,hemoglobin level, hemodynamic stability and use of inotropic support andComparison between dead and improved cases as regards age,diagnosis, hemoglobin . level,hemodynamic stability and use of inotropic support and need for blood transfusion . Mortality among critically ill children.

B. Overall

incidence:Incidence of pRBCs transfusion in critically ill children.

\section{Regression :}

A multivariate logistic regression model was used to identify independent risk factorsaffecting duration of admission and mortality in our PICU among these risk factors :diabetic ketoacidosis,age of studied patients in months, cardiac diseases, neurological diseases, respiratory diseases, post-operative admission, sepsis, hemoglobin level,hemodynamic un-stability, use of inotropic Support, frequency of pRBCs transfusion and indications of pRBCs transfusion including ( Anemia, Shock,

Shock and anemia).

D. Significance level:P value was considered significant if it was less than

(0.05).

E. Programs used and versions

Data was analyzed using STATA intercooled version 12.1. Quantitative 
data was represented as mean, standard deviation, median and range. Data was analyzed using student t-test to compare means of two groups. When the data was not normally distributed MannWhitney test was used. Qualitative data was presented as number and percentage and compared using either Chi square test or fisher exact test. Univariate and multivariate linear regression analyses were used to determine factors that affect the length of admission. Univariate and multivariate logistic regression analyses were used to determine factors that affect the mortality. Graphs were produced by using Excel or STATA program.

\section{STUDY RESULTS}

The study was conducted on $\mathbf{1 9 0}$ critically ill children of total $\mathbf{3 9 2}$ critically ill children admitted to PICU during one year.The mean age of studied critically ill children admitted to PICU was $\mathbf{1 9}$ month with $\mathrm{SD} \pm \mathbf{2 9}$ months , about $\mathbf{5 2 \%}$ was males. most of patients was diagnosed with respiratory and neurological diseases(about $\mathbf{2 5 \%}$ for each).the mean hemoglobin level on admission was $\mathbf{1 0} \mathrm{gm} / \mathrm{dl}$ with $\mathrm{SD} \pm \mathbf{2}$ and median $10 \mathrm{mg} / \mathrm{dl}$.about two thirds $\mathbf{7 1 \%})$ of studied patients was hemodynamically unstable and vasopressor support was used in treatment of $\mathbf{7 4 \%}$ of patients. About half $\mathbf{( 4 7 \% )}$ of the studied patients received pRBCs transfusion during their stay in PICU at least once.the mean duration of admission in PICU was $\mathbf{1 0}$ days with SD \pm 7 and median 8 days, and the mortality among studied cases was $\mathbf{3 9 \%}$.

$30 \%$ of patients received pRBCs for management of shock and $12 \%$ of patients received pRBCs for management of shock and anemia, while $4 \%$ of patients received pRBCs for management of anemia.non transfused cases was diagnosed mainly with cardiac and respiratory diseases $\mathbf{( 2 8 \% )}$ for each, while transfused cases was diagnosed mainly with neurological diseases $\mathbf{( 2 6 \% )}$ ) about half $\mathbf{( 5 2 \% )}$ of non-transfused cases was unstable ,while most

of transfused cases $(\mathbf{9 2 \%})$ was unstable . ( $\mathrm{P}$ value <0.0001).in non-transfused cases $\mathbf{7 4} \%$ of cases improved, while in transfused cases about $\mathbf{4 6 \%}$ of cases were improved, with a statistically significant difference between the two groups. ( $\mathrm{P}$ value $<\mathbf{0 . 0 0 0 1}$ ).

Most of cases who died washemodynamically unstable (97\%).About two thirds $\mathbf{( 7 4 \% )}$ of the patients who don't receive pRBCs improved and discharged from PICU, while more than half $\mathbf{5 4 \%}$ ) of the patients who received pRBCs died. ( $\mathrm{P}$ value $\mathbf{2 0 . 0 0 0 1 )}$. Correction of shock was the main indication of pRBCs transfusion as $\mathbf{4 2} \%$ of cases who died received PRBCs transfusion for correction of shock while $\mathbf{2 3 \%}$ of cases who improved received PRBCs transfusion for correction of shock. (P value $<\mathbf{0 . 0 0 0 1 )}$.

There was increased mortality with increasing frequency of transfusion as $75 \%$ of patients who received pRBCs transfusion twice died, $\mathbf{8 0 \%}$ of patients received pRBCs transfusion 3 times and all the patients who received pRBCs transfusion four times also died.

Respiratory diseaseswere the main risk factor that increase the duration of PICU stay ( $p$ value $\mathbf{0 . 0 0 1}$ ), while suffering from hemodynamic unstablity was the main risk factor for death in our PICU. ( $P$ value 0.02$)$ 


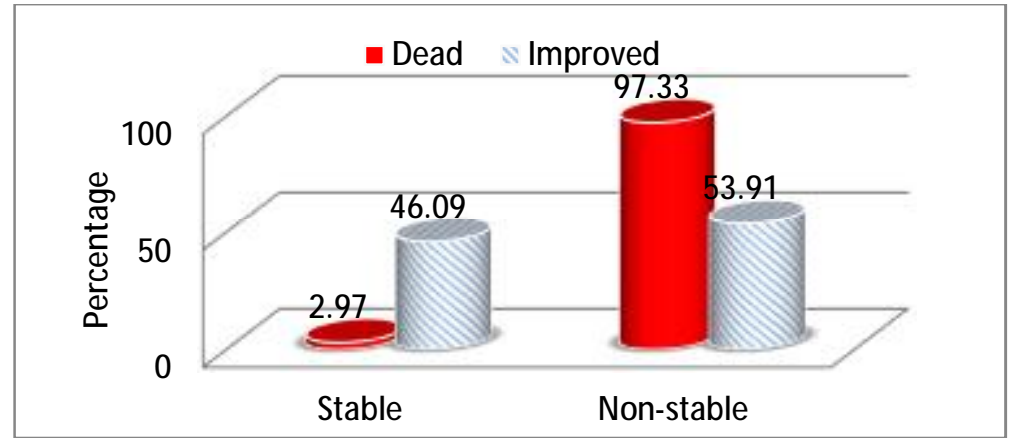

Figure (1): Comparison between dead and improved cases as regards hemodynamic stability

Table (II ) Multivariate logistic analysis of factors affecting mortality in PICU

\begin{tabular}{|l|c|c|}
\hline Variable & Adjusted Odds ratio (95\% confidence interval) & P value \\
\hline Age months & $0.99(0.97: 1.00)$ & 0.06 \\
\hline Cardiac & $0.36(0.15: 0.87)$ & 0.02 \\
\hline Non-stable & $25.15(1.86: 341.04)$ & 0.02 \\
\hline Support & $1.24(0.09: 17.72)$ & 0.87 \\
\hline Frequency & & \\
I & $0.92(0.28: 2.97)$ & 0.89 \\
2 & $3.16(0.70: 14.19)$ & 0.13 \\
3 & $4.17(0.36: 48.28)$ & 0.25 \\
4 & Omitted & \\
\hline Indication & & 0.81 \\
Anemia & $0.65(0.02: 19.82)$ & 0.89 \\
Shock & $0.92(0.30: 2.80)$ & 0.36 \\
Shock + anemia & $1.63(0.57: 4.72)$ & \\
\hline
\end{tabular}

Table ( I ) Multivariate regression analysis of factors affecting duration of admission (include significant variable in univariate analysis)

\begin{tabular}{|l|c|c|}
\hline Variable & $\begin{array}{c}\text { Regression co-efficient (95\% confidence } \\
\text { interval) }\end{array}$ & P value \\
\hline Respiratory & $3.76(1.48: 6.04)$ & 0.001 \\
\hline Non-stable & $2.30(-2.10: 6.69)$ & 0.30 \\
\hline Support & $-5.19(-9.58:-0.79)$ & 0.02 \\
\hline Frequency & $-9.16(-19.02: 0.70)$ & 0.07 \\
1 & $-2.71(-12.94: 7.53)$ & 0.60 \\
2 & $-5.18(-16.56: 7.53)$ & 0.37 \\
3 & $6.88(-2.86: 16.62)$ & 0.17 \\
\hline 4 & $8.05(-3.11: 19.21)$ & 0.16 \\
\hline Indication & $7.24(-2.73: 17.21)$ & 0.15 \\
Anemia & $6.51(-3.40: 16.43)$ & 0.20 \\
\hline Shock & & \\
Shock + anemia & & \\
\hline
\end{tabular}




$$
\text { mortality among studied cases. }
$$

\section{Discussion}

The mean age of studied cases was about 19 month, so younger children admitted to PICU are more than older children, a higher mean for age was found in study of Lacroix., et al 2007 andPierre., et al ,2015that difference could be explained by the vulnerability of small children for complications of infections as sepsis, congenital heart diseases and rapid deterioration of these cases especially in low socioeconomic locality. ${ }^{(2,6)}$

The main indication for admission in PICU was respiratory and neurological diseases mainly CNS infections and the mean Hemoglobin level on admission was $10 \mathrm{gm} / \mathrm{dl}$,this agreed with Pierre., et al ,2015

study, who found that male and female distribution nearlyhas the same percentage

that in our study, and the main indication for PICU admission was respiratory diseases followed by bacterial infections, viral infections.

In our study we found that almost half of the studied patients received $\mathrm{PRBC}$ transfusion during their stay in PICU at least once this disagree with in Pierre., et al ,2015who documented that $17 \%$ of the patients only received $\mathrm{PRBC}$ transfusion.

About $30 \%$ of patients received PRBCs for management of shock while $4 \%$ of patients received PRBCs for management of anemia while inPierre., et al 2015 study only $16 \%$ of patients received pRBCs for correction of shock and the main indication for transfusion was correction of anemia. ${ }^{(6)}$ The mean duration for admission in PICU was about 10 days withabout $\mathbf{4 0 \%}$
The study conducted byBateman., et al, 2008 was the first large, multicenter, prospective study of anemia, blood loss, and transfusion practices in critically ill children. anemia was a common problem, affecting $\mathbf{7 4 \%}$ of these children during or immediately before PICU admission, and about $\mathbf{5 0 \%}$ of patients received pRBCs during PICU admission, that's agreed with results of our study, also this study documented that Low $\mathrm{Hb}$ was the most common reason for transfusion ( $\mathbf{4 2 \%}$ of total transfusions) and this disagree with our study

Transfused cases was statistically compared with non-transfused cases. and we find that transfused critically ill children are younger than nontransfused cases this agree with findings in Bateman., et al, 2008 who found thatthe children who received a transfusion in the PICU were younger (mean age, 4.5 vs. 6.6 years for nontransfused cases (P value $\mathbf{0 . 0 0 1 )}{ }^{(7)}$ In our study we found that the mean hemoglobin level was $\mathbf{1 1}$ $\mathrm{gm} / \mathrm{dl}$ in non-transfused cases and 9 $\mathrm{gm} / \mathrm{dl}$ in transfused cases. Non transfused cases were diagnosed mainly with cardiac diseases and respiratory diseases (28\%) for each while transfused cases was diagnosed mainly with neurological diseases mainly CNS infections $\mathbf{( 2 6 \% )}$. about half $(\mathbf{5 2 \%})$ of non-transfused cases was unstable, while most of transfused cases $\mathbf{( 9 2 \% )}$ was unstable. these previous findings agreed with Armano., et al 2005, study that showed that a significant proportion of critically ill children receive at least one 
red blood cell transfusion during their PICU stay. presence of anemia, cardiac

disease, severe critical illness, and multiple organ dysfunction syndrome (MODS) are the most significant determinants of red blood cell transfusions in PICU. ${ }^{(8)}$

Transfused cases had shorter admission period than non-transfused cases which could be explained by increased mortality in transfused cases as unstability was the main indication for pRBCs transfusion. Previous results disagree with Pierre., et al ,2015who documented that transfused cases have longer admission periods and found that much lower percentage of un stable cases are found in both transfused and non-transfused groups than that of our study. The same study agree with results of our study that non-transfused cases was diagnosed mainly with respiratory diseases. also, transfused cases was diagnosed mainly with bacterial infection and the mean hemoglobin level was about $\mathbf{1 1} \mathrm{gm} / \mathrm{dl}$ in non-transfused cases and $9 \mathrm{gm} / \mathrm{dl}$ in transfused cases whish was nearly the same result found in our study. (6) Our study showed thatRespiratory diseaseswere the main risk factor that increase the duration of PICU stay ( $p$ value 0.001), while suffering from hemodynamic unstablity was the main risk factor for death in our PICU. (P value $\mathbf{0 . 0 2}$ )

- pRBCs transfusion following a restrictive blood transfusion strategy was safe in pediatric patients whose condition was stable in PICU, with no effect on mortality or duration of admission in PICU Our study results agreed with Lacroix., et al, 2007study who found that a restrictive strategy with a hemoglobin threshold of $7 \mathrm{~g}$ per deciliter resulted in a $96 \%$ reduction in the number of patients who had any transfusion exposure and a $\mathbf{4 4 \%}$ decrease in the number of red-cell transfusions administered, without increasing the rates of new or progressive MODS, in stable, critically ill children There were also no clinically important differences between the two groups in any secondary outcomes.

\section{${ }^{\text {(2) Our study has limitations: }}$}

It was conducted in a single center, which limits its external validity, the findings from one center may not apply to other centers with different patient populations . Selection of cases admitted to PICU strongly affected by limited resources, pediatric trauma patients not included, so most of study cases suffer from medical diseases .Our study was not able to highlight a cause-effect relationship between $\mathrm{pRBC}$ transfusions and outcomes in critically ill children only a randomized-controlled trial could establish such a causal link.

\section{Our study also has strengths :}

It's a prospective study with better data collection than retrospective studies andthe study included all consecutive PICU admissions over a 1-year period, which resulted in a case-mix with a limited risk of selection bias and no influence due to seasonal variation.

\section{Conclusion:}

Our study showed that respiratory diseases were the main risk factor that increase the duration of PICU stay ( $p$ value 0.001), while suffering from hemodynamic unstablity was the main risk factor for death in our PICU (P value 0.02) a restrictive blood transfusion strategy was safe in pediatric patients whose condition was stable in 
PICU, with no effect on mortality or durationof admission in PICU andthe studywas not able to highlight a causein critically ill children.

\section{Recommendations:}

Good care should be provided to smaller children and infants as this group is vulnerable, whilecarefull assessment and early correction of shock is the key point for improving mortality in PICU. be adopted in guidelines for management of critically ill children in PICU.

\section{Refferences}

1- Karam O, Tucci M, Ducruet $T$, Hume HA, Lacroix J, Gauvin F; Red blood cell

6,124-137.

2- Lacroix J, Hébert PC, Hutchison JS, Hume HA, Tucci M, Ducruet T, Gauvin F, Collet JP, Toledano BJ, Robillard P, Joffe A, Biarent D, Meert K, Peters MJ. Transfusion strategies for patients in pediatric intensive care unit, N. Engl. J. Med. 2007 ; 356(16), 1609-1619.

3- Morris KP, Naqvi N, Davies P, Smith M, Lee PW: A new formula for blood transfusion volume in the critically ill. Arch. Dis. Child. (2005). 90(7), 724728.

4- Jacques Lacroix, MD, FRCPC, FAAP, Pierre Demaret, MD, and Marisa Tucci, MD, FRCPC Red Blood Cell Transfusion: Decision Making in Pediatric Intensive Care Units, Seminars in Perinatology2012; Volume 36 , Issue 4

$225 \quad-\quad 231$

5-Ronald G. Strauss,BLOOD COMPONENT TRANSFUSIONS in

Nelson Textbook of Pediatrics 19th ,Robert M. Kliegman, MD Bonita F. Stanton, MD Joseph W. St. Geme III, effect relationship between RBC
transfusions and outcomes

neurological cases should receive good attention regarding nutritional status, management of anemia, and early detection and management of sepsis.

A restrictive blood transfusion strategy was safe in pediatric patients whose condition was stable in PICU, so it should

transfusion thresholds in pediatric patients with sepsis. Clin.Pediatr.Emerg. Med.

2005;volume

MD Nina F. Schor, MD, PhD Richard E. Behrman, MD ,Chapter 464 Red Blood Cell Transfusions and Erythropoietin Therapy, n e464-1,Elsevier Inc 2011. 66- Pierre Demaret, MD, MSc; Marisa Tucci, MD, FRCPC; Oliver Karam, MD, MSc; Helen Trottier, PhD; Thierry Ducruet, MSc; Jacques Lacroix, MD, FRCPC, PediatrCrit Care Med. 2015;16(6):505-514

7- Bateman ST, Lacroix J, Boven K, Forbes P, Barton R, Thomas NJ, Jacobs B, Markovitz B, Goldstein B, Hanson JH, Li HA, Randolph AG, Anemia, blood loss, and blood transfusions in North American children in the intensive care unit Am J RespirCrit Care Med. 2008 Jul 1;178(1):26-33. doi: 10.1164/rccm.2007 ; 11-1637OC

8- Armano, Ruth MD, FRCPC; Gauvin, France MD, MSc, FRCPC, FAAP; Ducruet, Thierry MSc; Lacroix, Jacques MD, FRCPC, FAAP ,November 2005 Volume 33 - Issue 11 - pp 2637-2644 Pediatric Critical Care( doi: 
SOHAG MEDICAL JOURNAL Vol. 21 No.1 Jan 2017
Characterization and outcome of packed red blood cells Hossam K. ALI.et al 